\title{
Exploratory Study of Signals for Asthma Drugs in Children, Using the EudraVigilance Database of Spontaneous Reports
}

\author{
Esmé J. Baan ${ }^{1}$ (1) - Veronique A. de Smet ${ }^{2} \cdot$ Christina E. Hoeve $^{1} \cdot$ Alexandra C. Pacurariu $^{1}$. \\ Miriam C. J. M. Sturkenboom ${ }^{4}$. Johan C. de Jongste ${ }^{3} \cdot$ Hettie M. Janssens ${ }^{3} \cdot$ Katia M. C. Verhamme ${ }^{1,2,5}$
}

Published online: 15 October 2019

(c) The Author(s) 2019

\begin{abstract}
Introduction As asthma medications are frequently prescribed for children, knowledge of the safety of these drugs in the paediatric population is important. Although spontaneous reports cannot be used to prove causality of adverse events, they are important in the detection of safety signals.

Objective Our objective was to provide an overview of adverse drug events associated with asthma medications in children from a spontaneous reports database and to identify new signals.

Methods Spontaneous reports concerning asthma drugs were obtained from EudraVigilance, the European Medicine Agency's database for suspected adverse drug reactions. For each drug-event combination, we calculated the proportional reporting ratio (PRR) in the study period 2011-2017. Signals in children (aged 0-17 years) were compared with signals in the whole population. Analyses were repeated for different age categories, by sex and by therapeutic area.

Results In total, 372,345 reports in children resulted in 385 different signals concerning asthma therapy. The largest group consisted of psychiatric events (65 signals). Only 30 signals were new, with seven, including herpes viral infections, associated with omalizumab. Stratification by age, sex and therapeutic area provided additional new signals, such as hypertrichoses with budesonide and encephalopathies with theophylline. Of all signals in children, 60 (16\%) did not appear in the whole population.

Conclusions The majority of signals regarding asthma therapy in children were already known, but we also identified new signals. We showed that signals can be masked if age stratification is not conducted. Further exploration is needed to investigate the risk and causality of the newly found signals.
\end{abstract}

Electronic supplementary material The online version of this article (https://doi.org/10.1007/s40264-019-00870-x) contains supplementary material, which is available to authorized users.

Esmé J. Baan

e.baan@erasmusmc.nl

1 Department of Medical Informatics, Erasmus Medical Centre, Erasmus University, Dr. Molewaterplein 50, 3015 GE Rotterdam, The Netherlands

2 Department of Pharmacy, Ghent University Hospital, Ghent, Belgium

3 Department of Pediatrics/Respiratory Medicine, Erasmus University/Sophia Children's Hospital, Rotterdam, The Netherlands

4 Julius Global Health, University Medical Centre Utrecht, Utrecht, The Netherlands

5 Department of Infection Control and Epidemiology, OLV Hospital, Aalst, Belgium

\section{Key Points}

Most safety signals regarding asthma therapy in children are known, but we identified several new signals that need to be explored further.

Lack of stratification by age and sex may mask safety signals in children.

\section{Introduction}

Asthma drugs are one of the most frequently used drugs in children, with approximately $21 \%$ of children aged $2-11$ years using asthma medication $[1,2]$. Since asthma is a chronic disease, this medication may be used for many years [3]. Like any drug, asthma medication may cause adverse 
reactions. Randomized controlled trials (RCTs) are the gold standard to investigate the efficacy of drugs but are limited in size and follow-up and therefore can only detect events that occur frequently and may miss rare or long-term side effects. Children remain underrepresented in RCTs, even though attempts have been made to increase these numbers, including the Paediatric Regulation that came into force in the EU in 2007 [4-7]. Given the lack of data, medication-including asthma drugs-is often not authorised for use in children, leading to substantial off-label use [8]. This is concerning because the efficacy and safety profile of drugs might differ between children and adults [9]. The risk assessment of drugs does not stop after market authorisation but should be monitored throughout the lifecycle of the drug [10]. Clearly, more child-specific safety and efficacy information on asthma drugs is needed, something the European Medicines Agency (EMA) emphasises [11].

Spontaneous report databases are an important source of safety information, and in fact remain the most frequent source from which regulatory action is initiated [12]. They cover large source populations, including special populations such as children, and may have more power to detect rare signals than mining of electronic healthcare records [13-15]. The EMA defines an adverse drug reaction as "a response to a medicinal product which is noxious and unintended" [16]. Spontaneous reports only contain information on the event and the drug of interest, not on the non-exposed events or exposure without events, so cannot be used to measure incidence rates. However, signal detection based on disproportionality analyses is still possible $[15,17,18]$. Since the risk of adverse events can be age specific, stratification of these analyses is recommended [19]. A previous study using EudraVigilance, the European database for suspected adverse reactions, characterised spontaneous reports of asthma drugs in children over the study period 2007-2011 [20]. The authors could not identify new signals in relation to asthma drugs, potentially because of low numbers. In this study, we aimed to investigate the safety of asthma drugs in children in EudraVigilance in the period 2011-2017. The objective of this study was to provide an overview of safety signals associated with asthma drugs in children and to identify new signals in the EudraVigilance database.

\section{Methods}

\subsection{Database}

We used the EudraVigilance database, which is developed and maintained by the EMA to collect reports of suspected adverse reactions. It contains adverse events reported in relation to any drug authorised or being studied in the European economic area. EudraVigilance contains spontaneous reports from healthcare professionals, patients and marketing authorisation holders and clinical trial safety data dating back to 1995 . The number of new reports in this database increases each year, with $1,238,178$ reports of adverse events collected in 2016 [21]. Events are coded using the Medical Dictionary for Regulatory Activities (MedDRA). For this study, information on the frequency of MedDRA (version 20.1) 'high-level terms' in spontaneous reports regarding asthma drugs was obtained. These terms are grouped by 'system organ classes' (SOCs), for example, by aetiology ('infections and infestations') or manifestation site ('respiratory, thoracic and mediastinal disorders') [22]. Drugs are coded by anatomical therapeutic chemical (ATC) classification.

\subsection{Data Selection}

All spontaneous reports submitted to EudraVigilance between 1 January 2011 and 1 January 2017 and concerning 24 asthma drugs were analysed. Reports concerning suspect or interacting single drugs or fixed-dose combinations from the following drug classes were included: $\beta_{2}$-adrenergic receptor agonists, inhaled corticosteroids (ICS), muscarinic receptor antagonist, chromones, xanthines, leukotriene receptor antagonists (LTRAs) and anti-immunoglobulin E (IgE) drugs (Table 1). Counts of spontaneous reports were provided for the whole paediatric group, by sex, by age category (0-2, 3-11 and 12-17 years) and for adults. Reports that did not include the patient's age were excluded. To calculate the measure of disproportionality, the background frequencies of adverse events for all drugs were also included in the data files. The free text narrative from the reports was not available because of data protection regulations. The retrieved data were at the group level only and included non-identifiable patient data, so no ethics review board approval was required.

\subsection{Signal Detection}

As one report may contain multiple drugs and/or multiple adverse events, we defined the combination of one drug and one event as drug-event combinations (DECs). To identify signals, we calculated the disproportionality in reporting using the proportional reporting ratio (PRR), defined as the proportion of the event of interest for a specific drug, with the comparator being the proportion of the event of interest for all other drugs from the database (other asthma and nonasthma drugs) [23]. The PRR was calculated only if a DEC was reported at least five times. We also calculated 95\% confidence intervals (CIs), and the criterion for a signal was a lower bound higher than one (PRR025 > 1) [19, 24-26]. We first calculated the PRR in reports concerning children only, then in the whole dataset including children and adults 
Table 1 Number of reports per drug in children

\begin{tabular}{llllc}
\hline ATC code & Drug name & Administration & Drug class & Reports $(N)$ \\
\hline R03DC03 & Montelukast & Oral & LTRA & 2210 \\
R03AC02 & Salbutamol & Inhaled & SABA & 947 \\
R03DX05 & Omalizumab & Subcutaneous & Monoclonal antibody & 690 \\
R03CC02 & Salbutamol, systemic & Oral, intravenous & SABA & 676 \\
R03BA02 & Budesonide & Inhaled & ICS & 584 \\
R03AK06 & Salmeterol+fluticasone & Inhaled & LABA+ICS & 565 \\
R03BA05 & Fluticasone & Inhaled & ICS & 391 \\
R03BA01 & Beclomethasone & Inhaled & ICS & 169 \\
R03AK07 & Formoterol+budesonide & Inhaled & LABA+ICS & 161 \\
R03DA04 & Theophylline & Oral, intravenous & Xanthine & 98 \\
R03CC03 & Terbutaline, systemic & Oral, intravenous & SABA & 87 \\
R03BB01 & Ipratropium bromide & Inhaled & SAMA & 85 \\
R03AC13 & Formoterol & Inhaled & LABA & 80 \\
R03AC03 & Terbutaline & Inhaled & SABA & 73 \\
R03BA08 & Ciclesonide & Inhaled & ICS & 36 \\
R03BC01 & Cromoglicic acid & Inhaled & Chromones & 34 \\
R03AC12 & Salmeterol & Inhaled & LABA & 32 \\
R03BA07 & Mometasone & Inhaled & ICS & 31 \\
R03DC01 & Zafirlukast & Oral & LTRA & 29 \\
R03AL01 & Fenoterol and ipratropium bromide & Inhaled & LABA + SAMA & 24 \\
R03AL02 & Salbutamol and ipratropium bromide & Inhaled & SABA + SAMA & 21 \\
R03AK10 & Vilanterol and fluticasone furoate & Inhaled & LABA + ICS & 16 \\
R03BB04 & Tiotropium bromide & Inhaled & LAMA & 9 \\
R03AK11 & Formoterol and fluticasone & Inhaled & LABA + ICS & 3 \\
\hline
\end{tabular}

$A T C$ anatomical therapeutic chemical, ICS inhaled corticosteroids, LABA long-acting $\beta$-agonist, LAMA long-acting muscarinic antagonist, LTRA leukotriene receptor antagonist, $S A B A$ short-acting $\beta$-agonist, SAMA short-acting muscarinic antagonist to examine masking of safety signals if stratification for children had not been done. We also repeated the analyses of paediatric reports while stratifying for age and sex [17]. Finally, we calculated another measurement of disproportionality-the PRR by therapeutic area (PRR-TA)—for paediatric reports. For this calculation, only reports related to asthma drugs for other events were used as the comparator. The PRR-TA has been proposed in the literature as a method to eliminate false signals due to confounding by indication [27].

\subsection{Definition of New Signals}

To identify already known signals, we searched the safety information as documented in the summary of product characteristics (SmPC). If the event was not listed in the SmPC, we also searched UpToDate and Martindale [28]. UpToDate is a widely used point-of-care clinical tool to support decision making that includes summaries of the current available evidence on medication, including possible adverse events [29-31]. Martindale is an online drug database published by the Pharmaceutical Press and is updated every 3 months
[32]. If an adverse event was not mentioned by any of these sources, we considered the signal on this adverse event to be a new signal.

\section{Results}

From January 2011 to January 2017, a total of 372,345 spontaneous reports on adverse events in children and 3,182,083 in adults were reported to EudraVigilance. From these reports, we identified 21,264 asthma medication-related DECs in children and 172,035 in adults for the same drugs. The asthma drug most often included in reports in children was montelukast (2210 reports), followed by inhaled salbutamol (947) and anti-IgE (omalizumab, 690) (Table 1). We identified 3697 unique DECs, 385 of which met the criteria of a safety signal in children. All safety signals and corresponding SOCs are listed in the Electronic Supplementary Material (ESM) 1. Signals were mainly related to the SOCs 'psychiatric disorders' $(n=65)$, 'respiratory, thoracic and mediastinal disorders' $(n=56)$ and 'nervous system disorders' $(n=29)$ (Fig. 1). Signals also often related to 
'Injury, poisoning and procedural complications' $(n=49)$, which included for example 'off-label uses' and 'accidental exposure to product'. We identified 31 signals of psychiatric adverse events in combination with montelukast, which also included the five most frequently reported DECs (Table 2). The highest PRR in children was for the association between montelukast use and 'vasculitides' (PRR 90.7; 95\% CI 46.3-177.9; 13 reports), followed by fluticasone and 'adrenal cortical hypofunctions' (PRR 59.5; 95\% CI 39.1-90.4; 22 reports) (Table 3 ). All ten signals with the highest PRR were already known.

\subsection{New Signals}

Most of the 385 signals in children were already known and thus described in the SmPC, UpToDate and/or Martindale; only 30 were classified as new. New signals most often concerned omalizumab, with seven new signals, followed by the fixed combination of salmeterol and fluticasone (six new signals) and montelukast (five new signals) (Table 4). See the ESM for a table presenting all signals not described in the SmPC but mentioned in UpToDate and/or Martindale.

\subsection{Proportional Reporting Ratio by Therapeutic Area}

The results of the PRR-TA differed from those of the overall PRR. Of all the signals, 126 disappeared when calculating the PRR-TA. This difference was most pronounced for 'respiratory, thoracic and mediastinal' disorders, as half of these signals disappeared when calculating the PRR-TA. On the other hand, the overall PRR of 38 DECs did not meet the definition of a safety signal, whereas the PRR-TA did show a signal. Three of these safety signals were new: omalizumab with 'bacterial infections NEC [not elsewhere classified]' and both omalizumab and budesonide with 'herpes viral infections' (Table 5).

\subsection{Children Versus All Ages}

Of the 385 signals in children, 60 (16\%) DECs no longer met the criteria of a signal when calculating the PRR in the whole dataset including all ages (both children and adults). All signals in children concerning the SOC 'respiratory, thoracic and mediastinal disorders' remained in the dataset that included children and adults together. In contrast, only 35 of the 50 signals in children concerning 'psychiatric disorders' remained in the whole dataset (Fig. 1).

\subsection{Stratification by Age}

Of all asthma medication-related DECs in children, 4429 (21\%) pertained to children aged 0-2 years, $11,319(53 \%)$ to children aged 3-11 years and 5516 (26\%) to children aged 12-17 years. Upon stratification by age, 42 additional safety signals appeared: 16 of these were in the age category 0-2 years, 14 in the category 3-11 years and 12 in the category $12-17$ years. These safety signals concerned 'pregnancy, puerperium and perinatal disorders' for events referring to in utero exposure and 'general disorders and administration site conditions', 'gastrointestinal disorders', 'psychiatric disorders' and 'nervous system disorders'. Six (14\%) of the signals that only appeared upon age stratification were classified as new (Table 6).

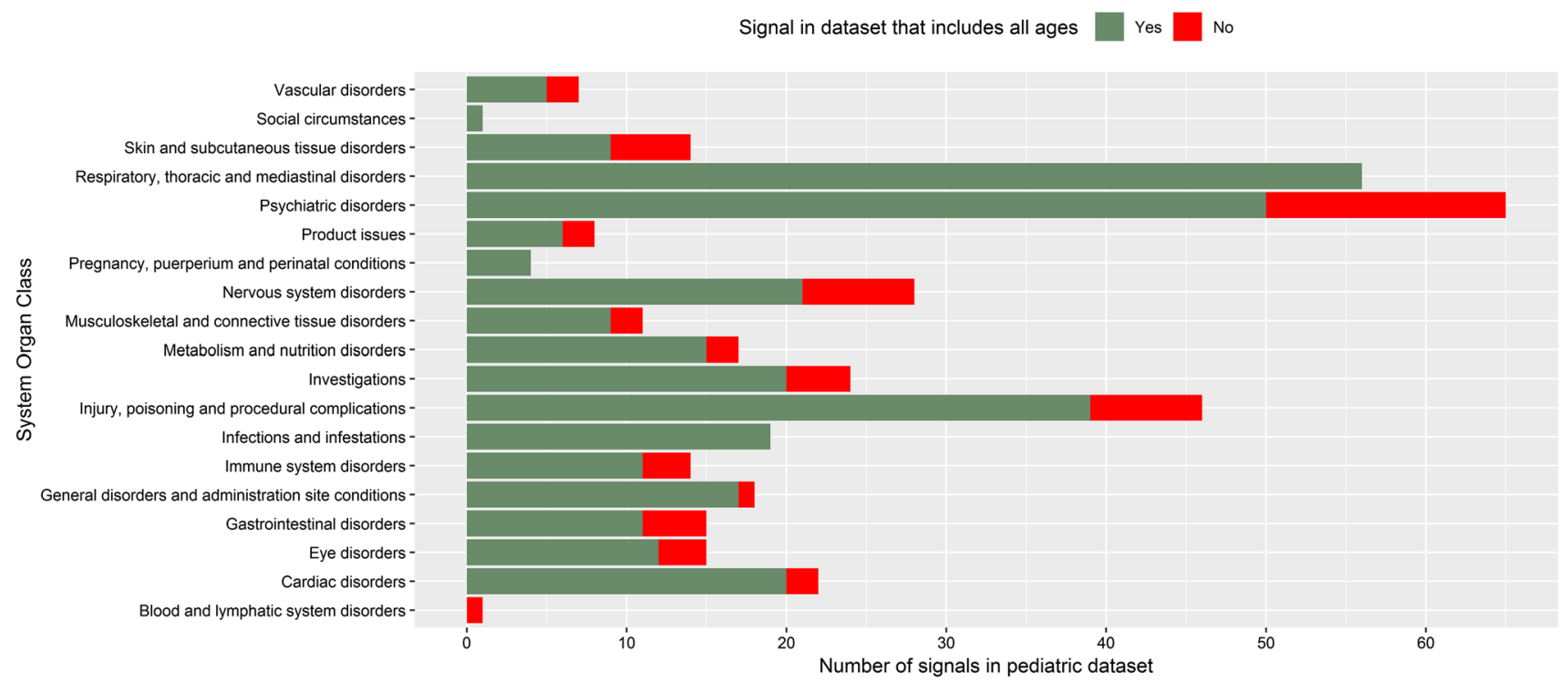

Fig. 1 Signals in children categorized as to whether it remains a signal in the whole dataset (children and adults) 
Table 2 Drug-event combinations of montelukast and psychiatric events
Table 3 Top ten strongest signals

\begin{tabular}{|c|c|c|}
\hline Reaction HLT & Reports $(N)$ & PRR $(95 \% \mathrm{CI})$ \\
\hline Behaviour and socialisation disturbances & 328 & $16.0(14.4-17.8)$ \\
\hline Parasomnias & 252 & $49.5(43.3-56.6)$ \\
\hline Anxiety symptoms & 249 & $6.4(5.7-7.2)$ \\
\hline Emotional and mood disturbances NEC & 247 & $6.9(6.1-7.7)$ \\
\hline Suicidal and self-injurious behaviour & 219 & $7.1(6.3-8.1)$ \\
\hline Abnormal behaviour NEC & 211 & $9.7(8.5-11.1)$ \\
\hline Disturbances in initiating and maintaining sleep & 163 & $10.4(8.9-12.1)$ \\
\hline Depressive disorders & 142 & $10.2(8.6-12.0)$ \\
\hline Fear symptoms and phobic disorders (including social phobia) & 72 & $27.5(21.5-35.1)$ \\
\hline Mood alterations with depressive symptoms & 67 & $8.0(6.3-10.2)$ \\
\hline Sleep disorders NEC & 66 & $7.0(5.5-9.0)$ \\
\hline Increased physical activity levels & 63 & $3.4(2.7-4.3)$ \\
\hline Speech articulation and rhythm disturbances & 61 & $6.3(4.9-8.1)$ \\
\hline Fluctuating mood symptoms & 59 & $20.6(15.8-26.9)$ \\
\hline Attention deficit and disruptive behaviour disorders & 33 & $14.8(10.4-21.0)$ \\
\hline Tic disorders & 32 & $8.1(5.7-11.5)$ \\
\hline Mood disorders NEC & 32 & $2.5(1.7-3.5)$ \\
\hline Obsessive-compulsive disorders and symptoms & 32 & $14.8(10.4-21.3)$ \\
\hline Panic attacks and disorders & 30 & $10.7(7.4-15.5)$ \\
\hline Thinking disturbances & 28 & $15.4(10.5-22.7)$ \\
\hline Confusion and disorientation & 25 & $2.0(1.3-2.9)$ \\
\hline Sleep disturbances NEC & 22 & $4.6(3.0-7.0)$ \\
\hline Affect alterations NEC & 21 & $9.3(6.0-14.4)$ \\
\hline Personality disorders NEC & 20 & $23.3(14.6-37.1)$ \\
\hline Psychotic disorder NEC & 18 & $3.2(2.0-5.1)$ \\
\hline Educational issues & 18 & $26.0(15.8-42.6)$ \\
\hline Psychiatric elimination disorders & 17 & $12.7(7.8-20.8)$ \\
\hline Criminal activity & 12 & $12.4(6.9-22.3)$ \\
\hline Anxiety disorders NEC & 10 & $14.8(7.8-28.3)$ \\
\hline Stereotypies and automatisms & 9 & $4.4(2.3-8.5)$ \\
\hline Impulse control disorders & 7 & $6.1(2.9-12.9)$ \\
\hline
\end{tabular}

$C I$ confidence interval, HLT high-level term, NEC not elsewhere classified, PRR proportional reporting ratio

\begin{tabular}{llcc}
\hline Name & Reaction HLT & Reports $(N)$ & PRR (95\% CI) \\
\hline Montelukast & Parasomnias & 252 & $49.5(43.3-56.6)$ \\
Salmeterol + fluticasone & Adrenal cortical hypofunctions & 30 & $57.4(39.9-82.6)$ \\
Fluticasone & Adrenal cortical hypofunctions & 22 & $59.5(39.1-90.4)$ \\
Budesonide & Adrenal cortical hypofunctions & 22 & $39.8(26.1-60.7)$ \\
Budesonide & Cataract conditions & 17 & $58.5(35.8-95.5)$ \\
Montelukast & Vasculitides & 13 & $90.7(46.3-177.9)$ \\
Salbutamol, inhaled & Thermal burns & 13 & $35.4(20.1-62.2)$ \\
Salmeterol+fluticasone & Glaucomas (excluding congenital) & 9 & $34.0(17.5-66.2)$ \\
Fluticasone & Cataract conditions & 7 & $34.1(16.2-72.1)$ \\
Fluticasone & Dental and periodontal infections and & 5 & $46.2(18.9-112.7)$ \\
& inflammations & & \\
\hline
\end{tabular}

CI confidence interval, HLT high-level term 
Table 4 All new signals by drug

\begin{tabular}{|c|c|c|c|c|}
\hline Reaction HLT & Reports & $\operatorname{PRR}(95 \% \mathrm{CI})$ & PRR-TA $(95 \%$ CI) & SOC \\
\hline \multicolumn{5}{|l|}{ Budesonide } \\
\hline Leukopenias NEC & 8 & $3.5(1.7-6.9)$ & $14.8(5.1-42.4)$ & Immune system disorders \\
\hline Hypertrichoses & 5 & $17.8(7.3-43.1)$ & $11.1(3.2-38.1)$ & Skin and subcutaneous disorders \\
\hline \multicolumn{5}{|l|}{ Fluticasone } \\
\hline Dyskinesias and movement disorders NEC & 13 & $2.3(1.3-3.9)$ & $1.3(0.7-2.2)$ & Nervous system disorders \\
\hline $\begin{array}{l}\text { Mental impairment (excluding dementia and } \\
\text { memory loss) }\end{array}$ & 5 & $2.8(1.2-6.6)$ & $1.3(0.5-3.2)$ & Nervous system disorders \\
\hline Hypoglycaemic conditions NEC & 5 & $2.9(1.2-7.1)$ & $2.7(1.0-6.8)$ & Metabolism and nutrition disorders \\
\hline \multicolumn{5}{|l|}{ Ipratropium bromide } \\
\hline Tremor (excluding congenital) & 6 & $6.6(3.1-14.3)$ & $2.2(1.0-4.7)$ & Nervous system disorders \\
\hline \multicolumn{5}{|l|}{ Montelukast } \\
\hline Paraesthesias and dysaesthesias & 43 & $2.0(1.5-2.7)$ & $1.5(1.0-2.2)$ & Nervous system disorders \\
\hline Skin injuries NEC & 15 & $2.2(1.3-3.7)$ & $1.8(0.9-3.6)$ & Skin and subcutaneous disorders \\
\hline Eyelid movement disorders & 10 & $3.9(2.1-7.2)$ & $5.5(1.7-17.4)$ & Eye disorders \\
\hline Lacrimation disorders & 6 & $2.4(1.1-5.3)$ & $1.1(0.4-2.9)$ & Eye disorders \\
\hline Panniculitides & 5 & $4.8(2.0-11.8)$ & $\mathrm{a}$ & Skin and subcutaneous disorders \\
\hline \multicolumn{5}{|l|}{ Omalizumab } \\
\hline Neurological signs and symptoms NEC & 38 & $1.6(1.2-2.2)$ & $2.7(1.9-3.8)$ & Nervous system disorders \\
\hline Feelings and sensations NEC & 27 & $2.5(1.7-3.6)$ & $2.5(1.6-3.8)$ & Nervous system disorders \\
\hline Visual disorders NEC & 11 & $2.0(1.1-3.6)$ & $2.0(1.1-3.9)$ & Eye disorders \\
\hline Speech and language abnormalities & 9 & $2.2(1.1-4.1)$ & $2.1(1.0-4.3)$ & Nervous system disorders \\
\hline Exfoliative conditions & 6 & $3.1(1.4-7.0)$ & $3.5(1.4-8.8)$ & Skin and subcutaneous disorders \\
\hline Sensory abnormalities NEC & 5 & $2.7(1.1-6.5)$ & $2.4(0.9-6.5)$ & Nervous system disorders \\
\hline Oral soft tissue pain and paraesthesia & 5 & $6.7(2.8-16.3)$ & $4.6(1.6-13.5)$ & Gastrointestinal disorders \\
\hline \multicolumn{5}{|l|}{ Salbutamol, inhaled } \\
\hline Non-site specific vascular disorders NEC & 9 & $3.9(2.0-7.5)$ & $4.8(2.0-11.4)$ & Vascular disorders \\
\hline Psychotic disorder NEC & 8 & $3.3(1.6-6.6)$ & $1.2(0.6-2.5)$ & Psychiatric disorders \\
\hline \multicolumn{5}{|l|}{ Salbutamol, systemic } \\
\hline Psychotic disorder NEC & 8 & $4.6(2.3-9.2)$ & $1.7(0.8-3.6)$ & Psychiatric disorders \\
\hline Non-site specific vascular disorders NEC & 6 & $3.6(1.6-8.1)$ & $3.8(1.5-9.7)$ & Vascular disorders \\
\hline \multicolumn{5}{|l|}{ Salmeterol + fluticasone } \\
\hline Visual disorders NEC & 10 & $2.2(1.2-4.2)$ & $2.3(1.2-4.4)$ & Eye disorders \\
\hline Increased intracranial pressure disorders & 9 & $4.3(2.2-8.2)$ & $4.1(1.9-8.8)$ & Nervous system disorders \\
\hline Confusion and disorientation & 7 & $2.2(1.0-4.5)$ & $1.7(0.8-3.8)$ & Psychiatric disorders \\
\hline Hypoglycaemic conditions NEC & 7 & $2.9(1.4-6.0)$ & $2.7(1.2-6.1)$ & Metabolism and nutrition disorders \\
\hline Tic disorders & 6 & $5.7(2.6-12.7)$ & $2.1(0.9-5.0)$ & Psychiatric disorders \\
\hline Memory loss (excluding dementia) & 5 & $3.1(1.3-7.4)$ & $2.1(0.8-5.5)$ & Nervous system disorders \\
\hline \multicolumn{5}{|l|}{ Theophylline } \\
\hline Encephalopathies NEC & 6 & $9.8(4.5-21.4)$ & $212.9(43.5-1041.5)$ & Nervous system disorders \\
\hline Abnormal behaviour NEC & 5 & $4.9(2.1-11.6)$ & $1.2(0.5-2.9)$ & Psychiatric disorders \\
\hline
\end{tabular}

$C I$ confidence interval, HLT high-level term, NEC not elsewhere classified, PRR-TA proportional reporting ratio by therapeutic area, SOC system organ class

aPRR-TA could not be calculated as the event was not reported in combination with other asthma drugs

\subsection{Stratification by Sex}

The sex of the patient was known in 20,726 DECs (98\%), with 11,926 (56\%) DECs concerning boys and $8800(41 \%)$ concerning girls. Upon sex stratification, $289(77 \%)$ signals remained. On the other hand, 30 DECs additionally met the definition of a safety signal, four of which (two each in males/females) were classified as new (Table 7). More signals appeared in boys only (97 signals) than in girls only (35 
Table 5 New signals unmasked by PRR-TA

\begin{tabular}{llllc}
\hline Drug & Reaction HLT & Reports (N) & PRR (95\% CI) & PRR-TA (95\% CI) \\
\hline Budesonide & Herpes viral infections & 9 & $1.0(0.5-1.9)$ & $7.7(3.3-17.9)$ \\
Omalizumab & Herpes viral infections & 8 & $0.8(0.4-1.5)$ & $5.3(2.2-12.5)$ \\
Omalizumab & Bacterial infections NEC & 6 & $0.8(0.4-1.8)$ & $13.8(3.9-48.9)$ \\
\hline
\end{tabular}

$C I$ confidence interval, $H L T$ high-level term, $N E C$ not elsewhere classified, $P R R$-TA proportional reporting ratio by therapeutic area

Table 6 New signals unmasked by stratification for age

\begin{tabular}{lllccc}
\hline Drug & Reaction HLT & Overall PRR (95\% CI) & Age group, years & $\begin{array}{c}\text { Reports in } \\
\text { age group }\end{array}$ & PRR in age group (95\% CI) \\
\hline Salbutamol, inhaled & Perception disturbances & $1.5(0.9-2.6)$ & $0-2$ & 6 & $14.8(6.6-33.0)$ \\
Salbutamol, inhaled & Cardiac septal defects congenital & $2.0(0.8-4.7)$ & $0-2$ & 5 & $2.8(1.2-6.6)$ \\
Budesonide & Neutropenias & $1.8(1.0-3.3)$ & $0-2$ & 5 & $3.6(1.5-8.5)$ \\
Theophylline & Febrile disorders & $1.1(0.7-1.7)$ & $3-11$ & 13 & $2.3(1.4-3.6)$ \\
Montelukast & Diabetes mellitus (including sub- & $1.6(0.9-3.0)$ & $3-11$ & 11 & $2.3(1.3-4.2)$ \\
& $\quad$ types) & & & 5 & $2.8(1.2-6.6)$ \\
Omalizumab & Cardiac signs and symptoms NEC & $1.2(0.7-2.1)$ & $3-11$ & 5 & $2.8(1.2-6.6)$ \\
Montelukast & Gastrointestinal atonic and hypomo- & $1.2(0.7-2.1)$ & $12-17$ & & 5 \\
& tility disorders NEC & & & & \\
\hline
\end{tabular}

$C I$ confidence interval, $H L T$ high-level term, $N E C$ not elsewhere classified, $P R R$ proportional reporting ratio

Table 7 New signals unmasked by stratification for sex

\begin{tabular}{llllll}
\hline Drug & Reaction HLT & Overall PRR (95\% CI) & Sex & Reports & PRR-sex (95\% CI) \\
\hline Fluticasone & Appetite disorders & $1.5(0.9-2.8)$ & Female & 7 & $2.5(1.2-5.1)$ \\
Montelukast & Alopecias & $1.5(0.8-2.8)$ & Male & 6 & $2.9(1.3-6.5)$ \\
Montelukast & Flatulence, bloating and distension & $1.3(0.7-2.5)$ & Female & 6 & $2.5(1.1-5.5)$ \\
Omalizumab & $\begin{array}{l}\text { Lid, lash and lacrimal infections, irrita- } \\
\text { tions and inflammations }\end{array}$ & $1.9(0.8-4.5)$ & Male & 5 & $3.5(1.5-8.5)$ \\
\hline
\end{tabular}

$C I$ confidence interval, $H L T$ high-level term, $N E C$ not elsewhere classified, $P R R$ proportional reporting ratio

signals). Signals more often appeared in boys than in girls for 'psychiatric disorders', 'musculoskeletal and connective tissue disorders' and 'eye disorders', whereas they appeared more often in girls for 'cardiac disorders' (Fig. 2).

\section{Discussion}

In this study, we investigated spontaneous reports of adverse events related to asthma drugs in children. We were able to include fivefold more reports than a previous analysis of EudraVigilance data from 2007 to 2011 [20]. We observed that spontaneous reports for montelukast were the most frequent, followed by reports for salbutamol, omalizumab and budesonide. Although $92 \%$ of the statistical signals were already known, we observed 30 new signals, especially for omalizumab. Calculation of the PRR by therapeutic area, age and sex revealed additional new signals, pointing to masking due to confounding by indication or effect modification.
Safety signals of asthma drugs were mainly related to psychiatric disorders, especially in combination with the use of montelukast. This is in line with a recent review that concluded that LTRAs are frequently associated with neuropsychiatric adverse events [6]. Awareness of the potential for psychiatric adverse events when initiating treatment with asthma drugs is important so that stopping treatment may be considered when the first symptoms of psychiatric events are observed. The second largest group of safety signals, 'respiratory, thoracic and mediastinal disorders', is of course prone to confounding by indication. This is further supported by our observation that half of the signals from this group disappeared when we calculated the PRR-TA.

Some observed new signals were already reported in the literature but not included in the SmPC, Martindale and UpToDate. For example, a study of spontaneous reports from Lareb, The Netherlands Pharmacovigilance Centre [33] associated hypertrichosis, both statistically and through causality assessment, with ICS, and cases of acute 


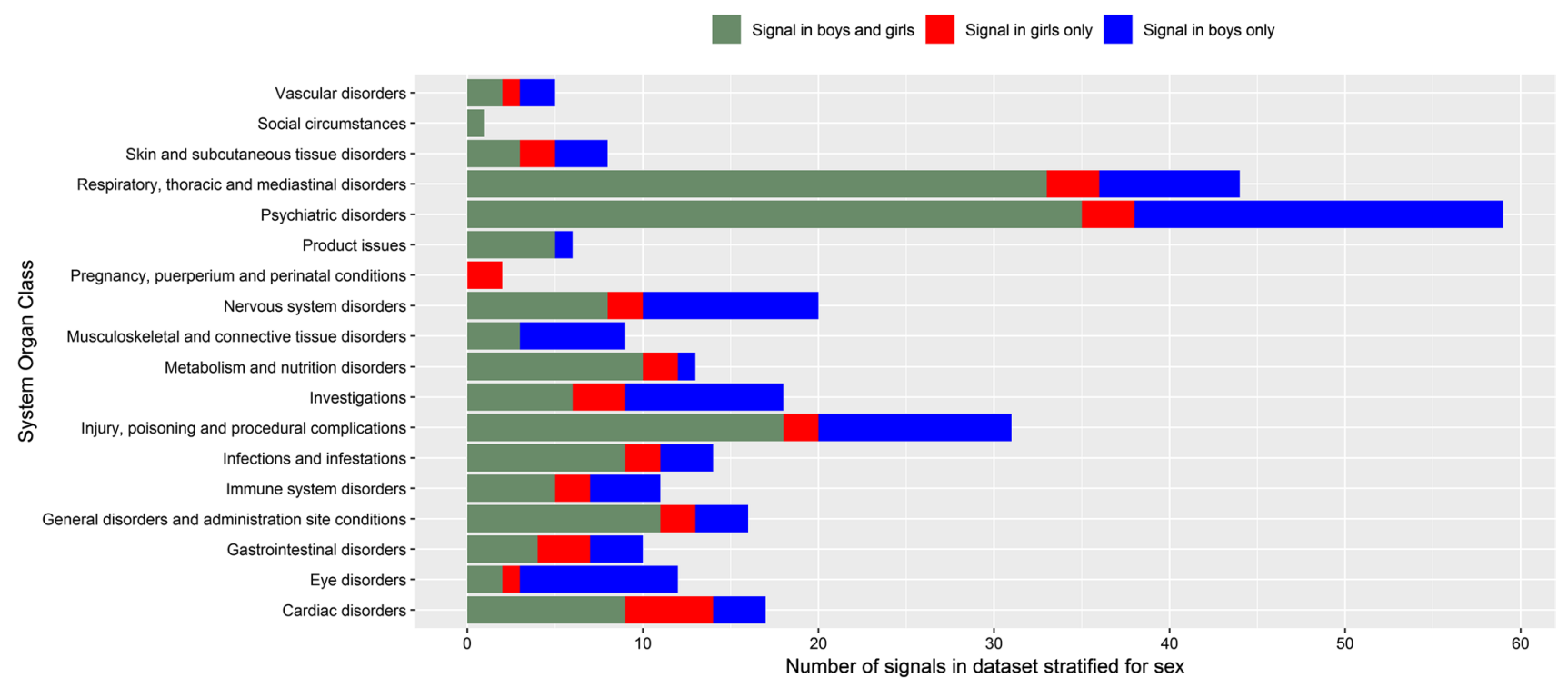

Fig. 2 Signals after stratification by sex categorized as a signal in boys only, girls only or both sexes

encephalopathies have been described with theophylline use. Although seizures are already listed as adverse reactions in the SmPC of theophylline, multiple cases of postictal coma lasting $>24 \mathrm{~h}$ have been described, regarding it as acute encephalopathy instead of a mere seizure [34]. We also found 'herpes viral infections' as a new signal associated with omalizumab. An increase in oral herpes in patients using omalizumab, without reaching statistical significance, has been mentioned in the literature [35]. One case of herpes labialis has been described in the literature, with the timing of herpes relapses directly related to omalizumab treatment [36]. Further studies should be conducted to confirm these new safety signals and investigate possible mechanisms.

Omalizumab, a monoclonal anti-IgE antibody, has been authorized in Europe since 2005. It is prescribed by specialist physicians to patients with asthma and IgE-mediated allergy that is difficult to control. Our results indicated new safety signals for omalizumab, including herpes viral infections, bacterial infections and visual disorders. The indication of use-namely, severe allergic asthma-implies that these patients were treated not only with omalizumab but also with other asthma drugs, especially high-dose ICS and systemic corticosteroids, both of which have immunosuppressive effects. Omalizumab is a systemic drug administered via intramuscular injection instead of inhaler therapy, which could also explain the relatively high number of reports.

We showed that many safety signals in children did not meet the criteria of a signal when calculating the PRR in the whole dataset that included adults. This means that such signals would have been missed had we not examined spontaneous reports in children separately. We also observed the importance of stratification for age within the paediatric group. Studies have demonstrated that stratification for age may unmask signals but adjustment for age does not improve the analyses [37, 38]. This emphasises the importance of actively monitoring the safety of drugs in children. When stratifying by sex, most signals remained. The prevalence of asthma, and thus the use of asthma drugs, is higher in boys than in girls. This may explain why more signals were reported for boys than for girls, as at least five reports are required for a signal [39].

The strength of our study lies in our use of one of the largest databases of spontaneous reports and data from many different countries, covering a heterogeneous paediatric population. This provided us with the opportunity to study the safety of a drug in real life and to report on signals of a relatively new drug, omalizumab. It also allowed us to stratify for age and sex. Spontaneous reports remain the most important source of detecting (new) safety signals, but the use and interpretation of these data has some limitations [14]. First, underreporting is likely in spontaneous report databases, and the adverse events that are reported are only the tip of the iceberg [40]. Another challenge of this type of study is that the MedDRA terms do not always match exactly the adverse reactions as mentioned in the SmPC and literature. This makes it difficult to interpret whether a signal is an already known adverse reaction. Reports might consist of a combination of multiple events and multiple drugs, and we were unable to deduct which was the culprit drug from these data, even though we only included those that were reported as suspected or interacting. Additional information from the report, such as the complete narrative, might have given more insight; unfortunately, data protection regulations 
meant these were unavailable. Also, some events were not reported frequently. This resulted in wide CIs, which require careful interpretation of the PRR. Finally, although we did have different combinations of medication and drugs, we did not have the number of reports containing a certain event combined with at least one type of asthma medication. This may have led to an underestimation of the true PRR-TA as the denominator was the number of combinations between the event and all asthma drugs.

For pharmacovigilance, it is paramount that any new suspected adverse drug reactions are reported immediately and include important details such as age and sex. This reporting is facilitated via online submission through the EudraVigilance website [41]. EudraVigilance also provides healthcare professionals with a graphical overview of the type of reported adverse drug reactions (by age, sex, type of reporter and outcome) of all centrally authorised drugs.

\section{Conclusion}

We characterized spontaneous reports of asthma drugs in children. We again showed the importance of investigating adverse events in children separately from adults and in different age and sex strata. We identified new signals that need to be further followed-up to investigate the true associations and causality.

\section{Compliance with Ethical Standards}

Funding No sources of funding were used to assist in the preparation of this study.

Conflict of interest Esmé J. Baan, Veronique A. de Smet, Christina E. Hoeve, Alexandra C. Pacurariu and Johan C. de Jongste have no conflicts of interest that are directly relevant to the content of this article. Hettie Janssens is involved in a study funded by Vectura with an unconditional research grant and has received a travel grant from Vertex, neither of which were related to the content of this work. Katia Verhamme works for a research group that has received unconditional research grants from Yamanouchi, Pfizer, Boehringer Ingelheim, Novartis and GSK, none of which was related to the content of this work. Miriam Sturkenboom led a research group that received research grants from GSK, Servier, Novartis; the GSK and Novartis studies were respiratory related. This study was independent. The views expressed in this article are the personal views of the authors and may not be understood or quoted as being made on behalf of or reflecting the position of the regulatory agency/agencies or organisations with which the authors are employed and/or affiliated.

Data sharing The data that support the findings of this study are available from the EMA, but restrictions apply to the availability of these data, which were used under license for the current study and so are not publicly available. A request for the data can be submitted to the EMA.

Ethical approval Ethical approval was not required for this study.
Open Access This article is distributed under the terms of the Creative Commons Attribution-NonCommercial 4.0 International License (http://creativecommons.org/licenses/by-nc/4.0/), which permits any noncommercial use, distribution, and reproduction in any medium, provided you give appropriate credit to the original author(s) and the source, provide a link to the Creative Commons license, and indicate if changes were made.

\section{References}

1. Sturkenboom MC, Verhamme KM, Nicolosi A, Murray ML, Neubert A, Caudri D, et al. Drug use in children: cohort study in three European countries. BMJ. 2008;337:a2245. https://doi. org/10.1136/bmj.a2245.

2. de Bie S, Coloma PM, Ferrajolo C, Verhamme KM, Trifiro G, Schuemie MJ, et al. The role of electronic healthcare record databases in paediatric drug safety surveillance: a retrospective cohort study. Br J Clin Pharmacol. 2015;80(2):304-14. https:// doi.org/10.1111/bcp.12610.

3. Global Initiative for Asthma. 2019 GINA report, Global strategy for asthma management and prevention. 2019. http://ginasthma. org/gina-reports/. Accessed 24 Sept 2019.

4. Joseph PD, Craig JC, Caldwell PH. Clinical trials in children. Br J Clin Pharmacol. 2015;79(3):357-69. https://doi.org/10.1111/ bcp. 12305.

5. Caldwell PH, Murphy SB, Butow PN, Craig JC. Clinical trials in children. Lancet. 2004;364(9436):803-11. https://doi. org/10.1016/S0140-6736(04)16942-0.

6. Leung JS, Johnson DW, Sperou AJ, Crotts J, Saude E, Hartling $\mathrm{L}$, et al. A systematic review of adverse drug events associated with administration of common asthma medications in children. PLoS One. 2017;12(8):e0182738. https://doi.org/10.1371/journ al.pone.0182738.

7. The European Parliament and the Council of the European Union. Regulation on medicinal products for paediatric use and amending Regulation (EEC) (EC No 1901/2006). 2006. https://www.ema. europa.eu/en/human-regulatory/overview/paediatric-medicines/ paediatric-regulation. Accessed 24 Sept 2019.

8. Cuzzolin L, Atzei A, Fanos V. Off-label and unlicensed prescribing for newborns and children in different settings: a review of the literature and a consideration about drug safety. Expert Opin Drug Saf. 2006;5(5):703-18. https://doi.org/10.1517/14740338.5.5.703.

9. Star K, Edwards IR. Pharmacovigilance for children's sake. Drug Saf. 2014;37(2):91-8. https://doi.org/10.1007/s4026 4-013-0133-8.

10. Guideline on good pharmacovigilance practices (GVP)—product- or population-specific considerations IV: paediatric population-EMA/572054/2016. [database on the Internet] 2018. https ://www.ema.europa.eu/en/human-regulatory/post-authorisation/ pharmacovigilance/good-pharmacovigilance-practices. Accessed 24 Sept 2019.

11. Human Medicines Research and Development Support. Inventory of paediatric therapeutic needs-respiratory (EMA/ PDCO/528943/2016). 2016. http://www.ema.europa.eu. Accessed 4 Aug 2018.

12. Pacurariu AC, Coloma PM, van Haren A, Genov G, Sturkenboom MC, Straus SM. A description of signals during the first 18 months of the EMA pharmacovigilance risk assessment committee. Drug Saf. 2014;37(12):1059-66. https://doi.org/10.1007/ s40264-014-0240-1.

13. Coloma PM, Trifiro G, Schuemie MJ, Gini R, Herings R, Hippisley-Cox J, et al. Electronic healthcare databases for active drug safety surveillance: is there enough leverage? Pharmacoepidemiol Drug Saf. 2012;21(6):611-21. https://doi.org/10.1002/pds.3197. 
14. Pacurariu AC, Straus SM, Trifiro G, Schuemie MJ, Gini R, Herings R, et al. Useful interplay between spontaneous ADR reports and electronic healthcare records in signal detection. Drug Saf. 2015;38(12):1201-10. https://doi.org/10.1007/s4026 4-015-0341-5.

15. Patadia VK, Schuemie MJ, Coloma P, Herings R, van der Lei J, Straus S, et al. Evaluating performance of electronic healthcare records and spontaneous reporting data in drug safety signal detection. Int J Clin Pharm. 2015;37(1):94-104. https://doi. org/10.1007/s11096-014-0044-5.

16. Quinto KB, Zuraw BL, Poon KY, Chen W, Schatz M, Christiansen $\mathrm{SC}$. The association of obesity and asthma severity and control in children. J Allergy Clin Immunol. 2011;128(5):964-9. https:// doi.org/10.1016/j.jaci.2011.06.031.

17. Dodd C, Pacurariu A, Osokogu OU, Weibel D, Ferrajolo C, Vo $\mathrm{DH}$, et al. Masking by vaccines in pediatric drug safety signal detection in the EudraVigilance database. Pharmacoepidemiol Drug Saf. 2018;27(11):1249-56. https://doi.org/10.1002/ pds.4623.

18. Alvarez Y, Hidalgo A, Maignen F, Slattery J. Validation of statistical signal detection procedures in eudravigilance postauthorization data: a retrospective evaluation of the potential for earlier signalling. Drug Saf. 2010;33(6):475-87. https://doi. org/10.2165/11534410-000000000-00000.

19. Committee for Medicinal Products for Human Use. Guideline on conduct of pharmacovigilance for medicines used by the paediatric population (EMEA/CHMP/PhVWP/235910/2005-rev.1). 2007.

20. Aagaard L, Hansen EH. Paediatric adverse drug reactions following use of asthma medications in Europe from 2007 to 2011. Int J Clin Pharm. 2014;36(6):1222-9. https://doi.org/10.1007/s1109 6-014-0020-0.

21. European Medicines Agency. 2016 Annual Report on EudraVigilance for the European Parliament, the Council and the Commission-16 March 2017 EMA/9942/2017. http://www.ema.europ a.eu. Accessed 24 Sept 2019.

22. Brown EG. Methods and pitfalls in searching drug safety databases utilising the Medical Dictionary for Regulatory Activities (MedDRA). Drug Saf. 2003;26(3):145-58. https://doi. org/10.2165/00002018-200326030-00002.

23. Evans SJ, Waller PC, Davis S. Use of proportional reporting ratios (PRRs) for signal generation from spontaneous adverse drug reaction reports. Pharmacoepidemiol Drug Saf. 2001;10(6):483-6. https://doi.org/10.1002/pds.677.

24. Waller P, van Puijenbroek E, Egberts A, Evans S. The reporting odds ratio versus the proportional reporting ratio: 'deuce'. Pharmacoepidemiology and drug safety. 2004;13(8):525-6. https://doi. org/10.1002/pds.1002 (discussion 7-8).

25. Blake KV, Saint-Raymond A, Zaccaria C, Domergue F, Pelle B, Slattery J. Enhanced paediatric pharmacovigilance at the european medicines agency: a novel query applied to adverse drug reaction reports. Paediatr Drugs. 2016;18(1):55-63. https://doi. org/10.1007/s40272-015-0154-0.

26. European Medicines Agency. Screening for adverse reactions in EudraVigilance (EMA/849944/2016). 2016. https://www.ema. europa.eu/en/documents/other/screening-adverse-reactions-eudra vigilance_en.pdf. Accessed 24 Sept 2019.
27. Grundmark B, Holmberg L, Garmo H, Zethelius B. Reducing the noise in signal detection of adverse drug reactions by standardizing the background: a pilot study on analyses of proportional reporting ratios-by-therapeutic area. Eur J Clin Pharmacol. 2014;70(5):627-35. https://doi.org/10.1007/s00228-014-1658-1.

28. UpToDate Inc. UpToDate. 2018. http://www.uptodate.com. Accessed 1 July 2018.

29. Isaac T, Zheng J, Jha A. Use of UpToDate and outcomes in US hospitals. J Hosp Med. 2012;7(2):85-90. https://doi.org/10.1002/ jhm.944.

30. Phua J, See KC, Khalizah HJ, Low SP, Lim TK. Utility of the electronic information resource UpToDate for clinical decisionmaking at bedside rounds. Singap Med J. 2012;53(2):116-20.

31. Sayyah Ensan L, Faghankhani M, Javanbakht A, Ahmadi SF, Baradaran HR. To compare PubMed Clinical Queries and UpToDate in teaching information mastery to clinical residents: a crossover randomized controlled trial. PLoS One. 2011;6(8):e23487. https://doi.org/10.1371/journal.pone.0023487.

32. Martindale: the complete drug reference [database on the Internet] 2019. Accessed 11 Apr 2019.

33. de Vries TW, de Langen-Wouterse JJ, de Jong-Van den Berg LT, Duiverman EJ. Hypertrichosis as a side effect of inhaled steroids in children. Pediatr Pulmonol. 2007;42(4):370-3. https://doi. org/10.1002/ppul.20589.

34. Saitoh M, Shinohara M, Ishii A, Ihara Y, Hirose S, Shiomi M, et al. Clinical and genetic features of acute encephalopathy in children taking theophylline. Brain Dev. 2015;37(5):463-70. https ://doi.org/10.1016/j.braindev.2014.07.010.

35. Lowe PJ, Georgiou P, Canvin J. Revision of omalizumab dosing table for dosing every 4 instead of 2 weeks for specific ranges of bodyweight and baseline IgE. Regul Toxicol Pharmacol. 2015;71(1):68-77. https://doi.org/10.1016/j.yrtph.2014.12.002.

36. Di Bona D, Fiorino I, Taurino M, Frisenda F, Minenna E, Pasculli C, et al. Long-term "real-life" safety of omalizumab in patients with severe uncontrolled asthma: A nine-year study. Respir Med. 2017;130:55-60. https://doi.org/10.1016/j.rmed.2017.07.013.

37. Osokogu OU, Dodd C, Pacurariu A, Kaguelidou F, Weibel D, Sturkenboom MC. Drug safety monitoring in children: performance of signal detection algorithms and impact of age stratification. Drug Saf. 2016;39(9):873-81. https://doi.org/10.1007/s4026 4-016-0433-x.

38. Star K, Sandberg L, Bergvall T, Choonara I, Caduff-Janosa P, Edwards IR. Paediatric safety signals identified in VigiBase: methods and results from Uppsala Monitoring Centre. Pharmacoepidemiol Drug Saf. 2019;28(5):680-9. https://doi.org/10.1002/ pds.4734.

39. Yung JA, Fuseini H, Newcomb DC. Hormones, sex, and asthma. Ann Allergy Asthma Immunol. 2018;120(5):488-94. https://doi. org/10.1016/j.anai.2018.01.016.

40. Hazell L, Shakir SA. Under-reporting of adverse drug reactions: a systematic review. Drug Saf. 2006;29(5):385-96. https://doi. org/10.2165/00002018-200629050-00003.

41. European Medicines Agency. http://www.adrreports.eu/en/repor t_side_effect.html. Accessed 24 Sept 2019. 